\title{
Launching Global Lung Function Initiative reference values in Belgium: tips and tricks
}

\author{
Eric Derom ${ }^{1}$, Giuseppe Liistro², Ellie Oostveen ${ }^{3}$, Eric Marchand ${ }^{4,5}$, \\ Lieven Bedert ${ }^{6}$, Rudi Peché ${ }^{7}$ and Wim Janssens ${ }^{8}$ on behalf of the Pulmonary \\ Function Working Group of the Belgian Thoracic Society
}

Affiliations: ${ }^{1}$ Dept of Respiratory Medicine, Ghent University Hospital, Ghent, Belgium. ${ }^{2}$ Service de Pneumologie, Cliniques Universitaires Saint-Luc, Institut de Recherche Expérimentale et Clinique (IREC), Pôle de Pneumologie, ORL and Dermatologie, Université Catholique de Louvain, Brussels, Belgium. ${ }^{3}$ Dept of Pulmonary Medicine, Antwerp University Hospital and University of Antwerp, Antwerp, Belgium. ${ }^{4}$ Service de Pneumologie, CHU-UCL-Namur, Site Godinne, Université catholique de Louvain, Yvoir, Belgium. ${ }^{5}$ Laboratoire de Physiologie Respiratoire, URPhyM, NARILIS, Université de Namur, Namur, Belgium. ${ }^{6}$ Dept of Respiratory Medicine, ZNA Middelheim Hospital, Antwerp, Belgium. ${ }^{7}$ Service de Pneumologie, Institut de Santé Publique du Pays de Charleroi, Université Libre de Bruxelles, Charleroi, Belgium. ${ }^{8}$ Dept of Chronic Diseases, Metabolism and Ageing (CHROMETA), University Hospital Leuven, KU Leuven, Leuven, Belgium.

Correspondence: Eric Derom, Dept of Respiratory Medicine, Ghent University Hospital - 7 K12 IE, De Pintelaan 185, B-9000 Ghent, Belgium. E-mail: eric.deromवugent.be

@ERSpublications

A model consisting of a motivated working group that identified the different stakeholders to convince them, through education, over the Global Lung Function Initiative reference values has appeared to be successful in implementing these in Belgium http://ow.ly/Q21630kuE1N

Cite this article as: Derom E, Liistro G, Oostveen E, et al. Launching Global Lung Function Initiative reference values in Belgium: tips and tricks. Eur Respir J 2018; 52: 1800922 [https://doi.org/10.1183/ 13993003.00922-2018].

Pulmonary function tests are pure physiological measurements. Spirometry allows the clinician to label lung diseases as either obstructive or restrictive, the latter requiring confirmation with static lung volume measurements. Transfer factor of the lung for carbon monoxide (TLCO) informs the clinician about the gas exchange properties of the respiratory system. A prerequisite to distinguish normal from abnormal data is the availability of accurate reference values as well as their lower and higher limits of normality. Choosing the correct set of reference values is crucial to discriminate health from disease in the individual and will influence any further medical processes. Indeed, results from one given subject may fall within the normal range using one equation while being abnormal using another [1-5]. The availability of accurate reference values is not only important in the diagnostic work-up and management of a single patient, it also leads to a better understanding of the global burden of lung disease, and allows a statistically correct estimation of the degree of impairment and disability in occupational medicine.

The 2005 American Thoracic Society (ATS)/European Respiratory Society (ERS) Task Force on Standardisation of Lung Function recommended for American subjects the ethnically appropriate National Health and Nutrition Examination Survey III reference equations for spirometry. It did not recommend any specific set of equations for use in Europe, being aware of the many shortcomings of the European Community for Steel and Coal equations still in use on that continent [6]. For TLCO, the ATS/ERS Task Force was unable to express any recommendation [6]. This prompted a number of clinicians, physiologists and researchers to establish the Global Lung Function Initiative (GLI) task force with the intention to collate existing data sources in order to create more robust and globally applicable reference equations. To this end, they took advantage of the newest statistical methods for analysing pulmonary function data across all ages [7]. In 2012, this task force published the multiethnic "all-age" reference values for 
spirometry [8]. Since then, these have been endorsed by the major European, American, Asian and Oceanian respiratory societies. More recently, the GLI network published the reference values for TLCO in Caucasians [4] and the same network is currently collecting data to establish reference equations for lung volumes.

Dissemination and publication of recommendations is often done by professional and national pulmonary societies, occasionally supported by industry, whereas their implementation is usually the responsibility of healthcare practitioners [9]. Neither the GLI, now an ERS Clinical Research Collaboration, nor the ERS are formally involved in the dissemination and implementation of the GLI reference values at the national level. Moreover, no strategies have been formulated on how to manage this endeavour. Convinced by the advantages of the GLI reference values, in 2013, the board of the Belgian Thoracic Society endorsed a working group to explore how the GLI reference values for spirometry could be introduced in Belgium, which eventually materialised in 2018. From the first discussions, the working group acknowledged that the introduction of the GLI reference values was a complex process that went far beyond a simple switch from one set of reference values to another. It also recognised that the transition should preferably occur on a nationwide basis within a limited time frame, to avoid confusion of healthcare providers and patients not familiar with reference values used in pulmonary function. The introduction of the GLI reference values would require teaching of pulmonologists on the advantages of the new equations, the concept of lower limit of normal and the attribution of Z-values to each measurement. Moreover, all stakeholders involved had to be identified as well the potential hurdles that could delay or interfere with a successful nationwide introduction of the GLI.

A master plan consisting of a three-stage approach was formulated and approved by the Belgian Thoracic Society board in 2013.

First, the pulmonologists were introduced to the GLI concept with posters during the 2014 winter meeting of the Belgian Thoracic Society. In addition, more detailed information on the "pros and cons" of GLI reference values were given to the majority of Belgian pulmonologists $(n=500)$ during the 18 local focus groups meetings. These focus groups, consisting of 20-25 pulmonologists each, are instructed by the Belgian healthcare authorities to gather at least four times a year to discuss issues related to quality and cost-effectiveness of healthcare. The working group urged these focus groups to invite one of its members to present the GLI concept, its advantages and the need for a collective transition. The same channel was used to convince paediatricians with specific interest in pneumology. Just before final launch, an update was given at the 2017 Belgian Thoracic Society meeting.

Second, the concept of the GLI was explained to lung function technicians during their 2013 and 2014 annual congress meetings by the late Prof. Quanjer. Updates on more technical aspects were provided at several regional meetings.

Third, from 2013 onwards, pulmonary function equipment manufacturers were contacted by members of the working group to prepare a collective transition. Technical barriers to upload the new reference values in older equipment and their financial repercussions were categorised, and estimations of the cost of updating the software were provided in all transparency. Eventually, all pulmonologists were regularly informed about the specific cost to update their equipment with the GLI reference values. This could vary between "completely free of charge", in the case of recently purchased equipment, and the need to order a completely new unit for pulmonary function in case the equipment was outdated. Often, however, an update of the driver for a personal computer, or other software, was sufficient. Even more challenging was to work out solutions to integrate the GLI in the electronic health records of one of the university hospitals, which are connected to seven other regional hospitals.

In 2017, the introduction of the GLI concept in Belgium was postponed until the reference values for TLCO became available, as it was estimated that the introduction of GLI for both values would be even more attractive to pulmonologists. Additionally, we developed and distributed a specific recommendation concerning the minimal requirements of a pulmonary function report (https://www.bvp-sbp.org). The pulmonologists were instructed to include absolute, median per cent predicted and Z-values on their report as well the source of the reference values. Starting in January 2018, the majority of pulmonologists were enthusiastic about the implementation and instructed for the transition. Since then, more and more hospitals have updated their software and implemented GLI as the standard reference in their protocol. Manufacturers told us that in rare cases, outdated equipment rendered installation of the update impossible, delaying it until the pulmonary function unit had been renewed in the upcoming year.

To the best of our knowledge, Belgium is the first country to have formally introduced the GLI reference values nationwide. It is expected that many other countries will follow. Different approaches going from a national survey (UK), to persistent debates of the pros and cons (France and Sweden), individual decisions 
whether to implement or not (the Netherlands and Spain), and university centres only (Germany, Italy and Lithuania) are currently seen. The "Belgian model" of a motivated working group that identified the different stakeholders to convince them through education for GLI appeared to be successful. A more challenging step is now to align primary care physicians with the new concepts of GLI.

Conflict of interest: E. Derom has nothing to disclose concerning the submitted manuscript, but received financial support from AstraZeneca, Chiesi and Boehringer Ingeheim to attend ERS and ATS congresses, and participated in national advisory boards for AstraZeneca, Boehringer, GSK, Novartis and Chiesi. W. Janssens reports receiving grants from GSK, AstraZeneca, Boehringer and Chiesi, outside the submitted work.

\section{References}

1 Kirkby J, Aurora P, Spencer H, et al. Stitching and switching: the impact of discontinuous lung function reference equations. Eur Respir J 2012; 39: 1256-1257.

2 Quanjer PH, Brazzale DJ, Boros PW, et al. Implications of adopting the Global Lungs Initiative 2012 all-age reference equations for spirometry. Eur Respir J 2013; 42: 1046-1054.

3 Quanjer PH, Weiner DJ. Interpretative consequences of adopting the Global Lungs 2012 reference equations for spirometry for children and adolescents. Pediatr Pulmonol 2014; 49: 118-125.

4 Stanojevic S, Graham BL, Cooper BG, et al. Official ERS technical standards: Global Lung Function Initiative reference values for the carbon monoxide transfer factor for Caucasians. Eur Respir J 2017; 50: 1700010.

5 Stanojevic S, Quanjer PH, Miller MR, et al. The Global Lung Function Initiative: dispelling some myths of lung function test interpretation. Breathe 2013; 9: 462-474.

6 Pellegrino R, Viegi G, Brusasco V, et al. Interpretative strategies for lung function tests. Eur Respir J 2005; 26 : 948-968.

7 Stanojevic S, Wade A, Stocks J, et al. Reference ranges for spirometry across all ages: a new approach. Am J Respir Crit Care Med 2008; 177: 253-260.

8 Quanjer PH, Stanojevic S, Cole TJ, et al. Multi-ethnic reference values for spirometry for the 3-95-yr age range: the global lung function 2012 equations. Eur Respir J 2012; 40: 1324-1343.

9 Grimshaw JM, Schunemann HJ, Burgers J, et al. Disseminating and implementing guidelines: article 13 in Integrating and coordinating efforts in COPD guideline development. An official ATS/ERS workshop report. Proc Am Thorac Soc 2012; 9: 298-303. 